\title{
Inclusión de harina, elaborada a partir de los frutos de Enterolobium Cyclocarpum y Brosimum Alicastrum como suplemento isocalórico e isoprotéico en la alimentación de ovinos
}

\section{Inclusion of flour, made from the fruits of Enterolobium Cyclocarpum and Brosimum Alicastrum as an isocaloric and isoproteic supplement in sheep feed}

\author{
DE LUNA-VEGA, Alicia, GARCÍA-SAHAGÚN, María Luisa, GONZÁLEZ-LUNA, Salvador y \\ PIMIENTA-BARRIOS, Enrique
}

Universidad de Guadalajara. Centro Universitario de Ciencias Biológicas y agropecuarias`(CUCBA).Camino Ramón
Padilla Sánchez 2100 Nextipac, 44600 Zapopan Jalisco

ID $1^{\text {er }}$ Autor: Alicia, De Luna-Vega

ID $1^{\text {er }}$ Coautor: María Luisa, García-Sahagún

ID $2^{\text {do }}$ Coautor: Salvador, González-Luna

ID $3^{\text {er }}$ Coautor: Enrique, Pimienta-Barrios

DOI: $10.35429 / J C P E .2019 .19 .6 .52 .61$

Recibido 09 Abril, 2019; Aceptado 28 Junio, 2019

\section{Resumen}

El estudio se realizó en el municipio de Cabo Corrientes localidad de Chacala, donde se encuentran distribuidas las especies en estudio en forma natural. Se realizaron análisis bromatológico del fruto de B. alicastrum y E. cyclocarpum como alternativa para mejorar la producción animal, al incrementar el consumo de proteína, energía y minerales en la dieta del ganado. Se realizó análisis bromatológico de proteína cruda (PC), extracto etéreo (EE) y extracto libre de nitrógeno (ELN) usando el método de Wendee (AOAC, 1990); fibra detergente neutra (FDN), fibra detergente ácida (FDA), celulosa, hemicelulosa y lignina (Van Soest y Wine, 1967), con el equipo ANKOM. Calcio y fósforo por colorimetría, potasio mediante la técnica del flamómetro (Van Soest, 1979); magnesio, azufre y cobre por el método de TMECC 04.06 / 4.13. La calidad nutritiva se analizó en materia seca, cenizas, grasa y fibra cruda. Se obtuvo diferencia significativa entre especies en proteína (22.90 vs 10.88), fibra (7.24 vs 13.18) y ELN donde el valor más alto fue para B. alicastrum $(67.02 \%)$ comparado con E. cyclocarpum $(60.45 \%)$. La inclusión de harina de los frutos de E. cyclocarpum y de B. alicastrum en las raciones alimenticias de los ovinos mejoró el consumo. El aumento de peso y la conversión alimenticia fueron mejores con la inclusión del $10 \%$. El valor económico de las raciones alimenticias con la inclusión de las harinas de los frutos de E. cyclocarpum y de B. alicastrum fue menor que el de la dieta testigo en ovinos estabulados, lo que permitió mantener su desarrollo con una disminución significativa de los costos de producción

Árboles Forrajeros, Rumiantes, Suplementación

\begin{abstract}
Study was performed in Chacala, Cabo Corrientes, where the species in study are widely distribuited in a natural form. Bromatologic analisis of fruit of $B$. alicastrum and $E$. Cyclocarpum were performed as an alternative to improve animal production, increasing protein consumption, energy and minerals on the livestock diet. Bromatologic analysis was made for crude protein $(\mathrm{CP})$, etereum extract (EE) and nitrogen free extract (NFE) with Wendee method (AOAC, 1990); neutral detergent fiber (NDF), acid detergent fiber (ADF), cellulose, hemicellulose and lignin (Van Soest and Wine, 1967) with ANKOM equipment. Calcium and phosphorus for colorimetry, potasium with flamometer technique (Van Soest, 1979); magnesium, sulphur and copper with TMECC 04.06 / 4.13 method. Nutritional quality was analized on dry matter, ashes, fat and crude fiber. Significative difference was obtained between species on protein (22.90 vs 10.88$)$, fiber (7.24 vs 13.18$)$ and NFE, were the higgest value was for B. alicastrum $(67.02 \%)$ compared with E. cyclocarpum $(60.45 \%)$. Inclussion of fruit flour from $E$. cyclocarpum and B. alicastrum on ovine alimentary rations improvement consumption. Weight increase and alimentary convertion were better with the $10 \%$ inclussion. Economic value of alimentary rations with inclussion of fruit flour of E. cyclocarpum and B. alicastrum was lower than witness diet on stabuled ovine, permiting maintain development with significative decrease of production costs.
\end{abstract}

Forage Trees, Ruminants, Supplementation

Citación: DE LUNA-VEGA, Alicia, GARCÍA-SAHAGÚN, María Luisa, GONZÁLEZ-LUNA, Salvador y PIMIENTA-BARRIOS, Enrique. Inclusión de harina, elaborada a partir de los frutos de Enterolobium Cyclocarpum y Brosimum Alicastrum como suplemento isocalórico e isoprotéico en la alimentación de ovinos. Revista de Energía Química y Física. 2019. 6-19: 52-61

\footnotetext{
* Correspondencia al Autor (Correo electrónico: dva20851@ cucba.udg.mx)

$\dagger$ Investigador contribuyendo como primer Autor
} 


\section{Introducción}

Entre las diferentes alternativas disponibles para reducir el deterioro ambiental producido por el auge expansionista de la ganadería tradicional extensiva en el trópico mexicano, se tiene la implementación de prácticas de tipo agroforestal, que impulsa la integración de árboles y arbustos con la producción animal y que podría dar la pauta para el desarrollo de sistemas de producción sustentable que no atente contra el frágil equilibrio ecológico del trópico mexicano, que incluso pudiera mejorar el comportamiento animal como ganancia de peso, consumo de alimento y conversión alimenticia, sin tener que depender de recursos externos (Laker, 1994).

Bajo la presión de producir alimentos en sistemas que mantengan estables su producción y rentabilidad a largo plazo, sin generar inequidad social $\mathrm{y}$ preservando todos los recursos naturales bajo el paradigma de la sostenibilidad, han cobrado especial importancia el uso de árboles forrajeros como fuente viable para la alimentación animal y más recientemente el manejo de sistemas silvopastoriles que integran el uso de pasturas, árboles y animales con diferentes objetivos y estrategias de producción (Giraldo, 1996a).

En México existe una gran variedad de especies arbóreas y arbustivas que tienen potencial par a ser incorporadas en los sistemas de producción en el trópico (Soto et al., 1997), los cuales podrían introducir elementos de sostenibilidad en los sistemas actuales (Enkerlin et al.,1997), al hacerlo menos dependiente de insumos externos, concentrados, energéticos y proteicos que tienen que ser adquiridos a costos elevados por los ganaderos (Laker, 1994).

La explotación pecuaria intensiva requiere de altas inversiones en las que el alimento generalmente representa entre el $60 \mathrm{y}$ $80 \%$ de los costos totales de producción (Bondi, 1988). Los principios nutritivos son compuestos químicos contenidos en los alimentos, que resultan necesarios para el mantenimiento, la reproducción, producción y salud de los animales. Los más importantes son: agua, carbohidratos, grasas, proteínas, minerales y vitaminas (Bondi, 1988). Las proteínas son necesarias en la alimentación animal por las numerosas funciones que desarrollan en el organismo animal.

\section{Objetivo general}

Por lo cual dentro de los objetivos del presente trabajo es evaluar aspectos nutritivos del fruto de estas especies arbóreas para incorporarlas a los sistemas de explotación pecuaria como una alternativa para mejorar el rendimiento animal, al incrementarse el consumo de proteína, energía y minerales en la dieta del ganado a un costo reducido

\section{Objetivos particulares}

1. Determinar la calidad nutritiva de la harina de los frutos de E. cyclocarpum y B. alicastrum (MO), proteína cruda (PC), fibra detergente neutra (FDN), fibra detergente ácida (FDA) de los frutos de E. cyclocarpum y B. alicastrum

2. Evaluar el comportamiento de ovinos al incluir la harina de frutos de $E$. cyclocarpum y B. alicastrum en su dieta alimenticia

3. Estimar el valor económico de las raciones en estudio

\section{Materiales y métodos Localización}

El estudio se realizó en el municipio de Cabo Corrientes en la localidad de Chacala, donde se encuentran ampliamente distribuidas las especies en estudio dentro de los tipos de vegetación de selva mediana subcaducifolia. La Comunidad se ubica a $31 \mathrm{~km}$ al Noroeste de la población de El Tuito cabecera municipal de Cabo Corrientes, Jal., en las coordenadas $20^{\circ} 10^{\prime} 55^{\prime}$ ', a los $20^{\circ} 31^{\prime} 00^{\prime \prime}$ de latitud norte y de los $105^{\circ} 10^{\prime} 00^{\prime \prime}$ a los $105^{\circ} 41^{\prime} 25^{\prime \prime}$ de longitud oeste. El municipio limita al norte con el de Puerto Vallarta y el Océano Pacífico, al sur con el de Tomatlán, al oriente con el de Talpa de Allende y al poniente con el Océano Pacífico.

\section{Aspectos nutricionales de las especies en estudio}

La determinación de la composición química de los frutos se realizó en el laboratorio de nutrición animal, del Departamento de Producción Animal, de la División de Ciencias Veterinarias, para medir las fracciones químicas de los frutos por análisis bromatológico de proteína cruda (PC), extracto etéreo (EE) y extracto libre de nitrógeno (ELN)) usando el método de Wendee (AOAC, 1990). 
Se determinaron las fracciones de fibra detergente neutra (FDN), fibra detergente ácida (FDA), celulosa, hemicelulosa y lignina (técnica de Van Soest y Wine, 1967), con el equipo ANKOM. Minerales. El calcio y fósforo fueron analizados por métodos colorimétricos, el potasio se analizó mediante la técnica del flamómetro (Van Soest, 1979), magnesio, azufre y cobre por el metodo de TMECC 04.06 / 4.13 Prueba de comportamiento. Se llevó a cabo una prueba de comportamiento con ovinos pelibuey en la cual se evaluó el consumo de alimento, aumento de peso y conversión alimenticia, además de un análisis económico con porcentajes de 10 y $20 \%$ de inclusión de harina de los frutos en estudio más las dietas testigo ( $0 \%$ de harina de frutos).

\section{Tratamientos estudiados}

El trabajo experimental consistió en evaluar tres tratamientos con los porcentajes de inclusión de 0,10 y $20 \%$ de harina de E. cyclocarpum y de $B$. alicastrum en dietas isocalóricas $\mathrm{e}$ isoprotéicas. $\mathrm{T}_{1} \mathrm{~T}_{4}=0 \%, \mathrm{~T}_{2} \mathrm{~T}_{5}=10 \%, \mathrm{~T}_{3} \mathrm{~T}_{6}=$ $20 \%$, para cada una de las harinas de los frutos arbóreos. Los ingredientes y análisis bromatológicos de ingredientes y mezclas se muestran en los Tablas del 1 al 3.

\begin{tabular}{|l|r|r|r|r|r|}
\hline & \multicolumn{2}{|c|}{ Testigos } & \multicolumn{2}{c|}{ E. cyclocarpum } & \multicolumn{2}{|c|}{ B. alicastrum } \\
\hline Ingredientes & & $10 \%$ & $20 \%$ & $10 \%$ & $20 \%$ \\
\hline Rastrojo de maíz & 27.00 & 54.50 & 27.00 & 54.00 & 27.00 \\
\hline Alfalfa & 12.00 & - & 17.40 & - & 13.20 \\
\hline Trigo & 22.00 & - & 4.80 & - & 4.80 \\
\hline Sorgo & 20.00 & 17.00 & 15.00 & 15.00 & 15.00 \\
\hline Melaza & 12.10 & 10.00 & 10.00 & 10.00 & 10.00 \\
\hline Harina de pescado & 2.00 & 3.20 & 1.00 & 5.70 & 5.20 \\
\hline E. cyclocarpum & - & 10.00 & 20.00 & - & - \\
\hline B. alicastrum & - & - & - & 10.00 & 20.00 \\
\hline Roca fosfórica & 1.00 & 1.00 & 1.00 & 1.00 & 1.00 \\
\hline Carbonato de calcio & 1.00 & 1.00 & 1.00 & 1.00 & 1.00 \\
\hline Vitaminas* & 0.25 & 0.25 & 0.25 & 0.25 & 0.25 \\
\hline Minerales* & 0.05 & 0.05 & 0.05 & 0.05 & 0.05 \\
\hline Sal & 1.00 & 1.00 & 1.00 & 1.00 & 1.00 \\
\hline Urea & 1.60 & 2.00 & 1.50 & 2.00 & 1.50 \\
\hline Totales & 100.00 & 100.00 & 100.00 & 100.00 & 100.00 \\
\hline
\end{tabular}

Tabla 1 Porcentajes de E. cyclocarpum y B. alicastrum e ingredientes utilizados en cada tratamiento, por dieta

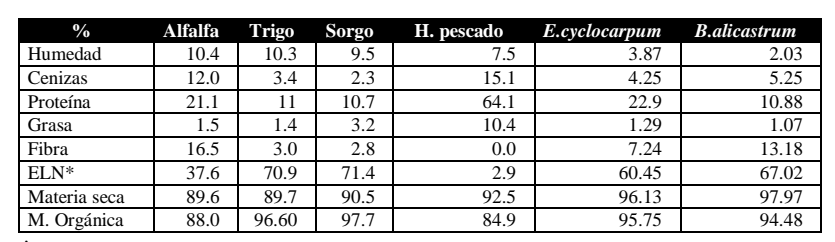

*Elementos libres de nitrógeno (ELN)

Tabla 2 Análisis bromatológico de los ingredientes incluidos en los tratamientos de la harina de frutos de $E$. cyclocarpum y B. alicastrum, reportado en $\%$

\begin{tabular}{|l|r|r|r|r|r|}
\hline & \multicolumn{1}{|c}{ Testigos } & \multicolumn{2}{c|}{ E. cyclocarpum } & \multicolumn{2}{c|}{ B. alicastrum } \\
\hline Concepto & $0 \%$ & $10 \%$ & $20 \%$ & $10 \%$ & $20 \%$ \\
\hline Humedad & 10.50 & 10.00 & 10.30 & 10.51 & 11.20 \\
\hline Cenizas & 10.60 & 10.10 & 10.15 & 10.31 & 10.30 \\
\hline Proteína & 15.90 & 15.40 & 15.43 & 15.46 & 15.43 \\
\hline Grasa & 1.40 & 1.40 & 1.45 & 1.20 & 1.00 \\
\hline Fibra & 10.60 & 10.10 & 15.18 & 10.29 & 10.30 \\
\hline ELN* & 51.00 & 53.00 & 47.49 & 52.23 & 51.77 \\
\hline Materia seca & 89.50 & 90.00 & 89.70 & 89.49 & 88.80 \\
\hline Materia orgánica & 89.40 & 89.90 & 89.85 & 89.69 & 89.70 \\
\hline
\end{tabular}

* Elementos libres de nitrógeno (ELN)

Tabla 3 Análisis bromatológico de los tratamientos estudiados

\section{Materiales utilizados}

En la presente investigación se utilizó una nave para ovinos con una dimensión de $10 \mathrm{~m}$ de ancho por $40 \mathrm{~m}$ de largo, con techo de lámina aislante, piso de cemento con las ventanas hacia el norte y hacia el sur, el enjarre es de cemento pintado de blanco.

En esta nave se lotificaron 24 corrales de dos metros de ancho $\mathrm{x}$ tres de largo, cada uno separado con tubos de fierro con un diámetro de tres pulgadas. Para el suministro de agua y alimento se utilizaron comederos y bebederos de plástico. Se dispuso de dos básculas de precisión, una con capacidad de $2.600 \mathrm{~kg}$ para pesar los minerales y la otra de $120 \mathrm{~kg}$ para pesar los ovinos y los ingredientes de los tratamientos, así como un molino de martillos con una criba de dos $\mathrm{mm}$ y una revolvedora horizontal para moler y mezclar los ingredientes de cada ración. Se utilizaron un total de 72 ovinos de la raza Pelibuey con un peso promedio de $25 \mathrm{~kg}$, vacunada y desparasitados interna $y$ externamente.

\section{Procedimiento experimental:}

Los frutos de E. cyclocarpum y B. alicastrum fueron secados al sol durante ocho días consecutivos y después molidos en un molino de martillo, la mezcla de los ingredientes se hizo en una revolvedora horizontal. Un total de seis tratamientos con cuatro repeticiones (tres animales por repetición y 24 por tratamiento) fueron estudiados bajo un diseño experimental de bloques al azar, boqueado por el peso de los animales en; ligeros (l) $n=12$, con un peso promedio de $24 \mathrm{~kg}$ medianos $(\mathrm{m}) \mathrm{n}=12$ peso promedio $25 \mathrm{~kg}$ y pesados $(\mathrm{p}) \mathrm{n}=12$ peso promedio $26 \mathrm{~kg}$. El peso promedio de los animales por tratamiento fue de $25 \mathrm{~kg}$. 
Cada animal se aretó con un número progresivo, después fueron distribuidos en los diferentes tratamientos. Se desparasitó con ivermectina (Ivomec), y se vacunó contra las enfermedades más comunes en la zona (septicemia hemorrágica, carbón sintomático y edema maligno) aplicando Bacterina Triple y contra Ántrax. Después de distribuidos los animales, se les dio agua para evitar la deshidratación. Tuvieron un periodo de 8 días de adaptación para las nuevas dietas en estudio.

El experimento tuvo una duración de 72 días, durante el cual se registraron las siguientes mediciones:

a. Ganancia peso. Los animales se pesaron al inicio del experimento y después a intervalos regulares cada 14 días, con un ayuno previo de 24 horas de alimento y agua. Las ganancias de peso catorcenal se obtuvieron por diferencia entre dos pesadas consecutivas.

b. Consumo de alimento. El agua fue ofrecida ad libitum. El alimento se pesaba y se ofrecía el $50 \%$ a las 9 de la mañana y $50 \%$ a las 6 de la tarde; al día siguiente a las 8 de la mañana se recolectaban el alimento rechazado, se pesaba y por diferencia se sacó el consumo diario.

Los parámetros a medir fueron:

- $\quad$ Peso diario de alimento proporcionado a los ovinos

- Peso del alimento rechazado diariamente

- Peso corporal de los ovinos catorcenalmente, después de una dieta de 24 horas y análisis económico de las raciones

- Los parámetros indirectos fueron:

- Kg de alimento consumido por día

- Ganancia media diaria de peso

- Conversión alimenticia

\section{Diseño experimental}

El experimento se desarrolló bajo un diseño de bloques al azar, bloqueado por el peso de los animales en; ligero (l), mediano (m) y pesado (p) El modelo estadístico utilizado fue: $\mathrm{Yij}+\mathrm{M}+$ $\mathrm{Ti}+\mathrm{Bj}+\mathrm{TiBj}$
Dónde:

Yij $=$ Variable de respuesta

$\mathrm{M}=$ Media ponderada

$\mathrm{Ti}=$ Efecto de tratamiento i-esimo

$\mathrm{Bj}=$ Efecto del bloque $\mathrm{j}$-iesimo

$\mathrm{Ti} \mathrm{Bj}=$ Interacción entre tratamiento i-esimo y

bloque j-iesim

Variables bajo estudio

Ganancia de peso

Consumo de alimento

Conversión alimenticia

Los resultados fueron analizados mediante ANOVA. Utilizando los procedimientos del paquete estadístico STATISCA 6.0, se hizo la comparación de medias con la prueba de Tukey, $(\mathrm{P}<0.05)$.

\section{Resultados y discusión}

\section{Aspectos nutricionales de las especies arbóreas en estudio}

La calidad nutritiva en cuanto a MS, PC, MO, ELN, humedad, cenizas, grasa y fibra cruda. La única diferencia significativa encontrada entre las especies en estudio correspondió a la proteína (22.90 vs 10.88), fibra (7.24 vs 13.18) y elementos libres de nitrógeno (ELN) (60.45 vs $67.02 \%$ ) (Tabla 4).

ELN está representado principalmente por los azúcares solubles, de muy fácil digestión. EL valor más alto fue $B$. alicastrum $(67.02 \%)$ comparado con el E. cyclocarpum (60.45\%).

El ELN en estos frutos es de suma importancia para el balance de energía y proteína en el rumen, ya que representa una fuente de energía fácilmente fermentable, la cual es aprovechada rápidamente por la flora microbiana para todos los procesos de síntesis. Corroborando lo publicado por Bondi, 1988, con el contenido de ELN de estos frutos estamos proporcionando a los rumiantes un fuente de energía para la flora microbiana, que esta a su vez proporcionará al animal, mayor capacidad de digestión y aprovechamiento de los nutrientes. 


\begin{tabular}{|l|r|r|}
\hline Determinación & \multicolumn{1}{c}{$\begin{array}{c}\text { E. } \\
\text { cyclocarpum }\end{array}$} & $\begin{array}{c}\text { B } \\
\text { alicastrum }\end{array}$ \\
\hline Humedad & 3.87 & 2.03 \\
\hline Cenizas & 4.25 & 5.52 \\
\hline Proteína & 22.90 & 10.88 \\
\hline Grasa & 1.29 & 1.07 \\
\hline Fibra & 7.24 & 13.18 \\
\hline ELN & 60.45 & 67.02 \\
\hline Materia seca & 96.13 & 97.97 \\
\hline FDN & 34.58 & 19.37 \\
\hline FDA & 31.28 & 17.25 \\
\hline Lignina & 25.66 & 12.25 \\
\hline Celulosa & 5.26 & 4.25 \\
\hline Hemicelulosa & 3.30 & 2.87 \\
\hline Nitrógeno \% & 3.66 & 1.74 \\
\hline Fósforo \% & 0.30 & 0.20 \\
\hline Potasio \% & 0.86 & 1.00 \\
\hline Calcio \% & 0.27 & 0.20 \\
\hline Magnesio \% & 0.78 & 0.76 \\
\hline Azufre \% & 0.22 & 0.24 \\
\hline Cobre ppm & 5.56 & 5.52 \\
\hline
\end{tabular}

Elementos libres de nitrógeno (ELN).

Fibra detergente neutro (FDN), fibra detergente ácida (FDA)

Tabla 4 Análisis químico proximal de la harina de los frutos de E. cyclocarpum y B. alicastrum reportados en porcentaje

El contenido de proteína cruda (PC) obtenido en B. alicastrum (10.88\%) resulto más bajo de lo esperado comparado con otras investigaciones entre ellos Sosa et al., 2004 (13.81); Palma, 2006 (13.04\%), señalaron que la proteína varía del 12 al 20\%; sin embargo Carranza et al., 2003 que reportaron $8.65 \mathrm{y}$ $9.68 \%$ valores más bajos encontrados en este trabajo. Para el E. cyclocarpum, (Carranza et al. 2003; Cecconello et a., 2003), reportaron 18.28 y $14.10 \%$, teniendo una variación con los valores reportados por parte de Álvarez et al., 2003, reportó en PC 17.2\%, Palma en el 2006 encontró el $15.49 \%$ valores más bajos a los encontrados en este trabajo.

Esta variación se puede deber que estos autores sus investigaciones fueron realizados en diferente estados y diferentes épocas. Por su parte Barrientos (2006) reportó para la harina del fruto E. cyclocarpum valores de $27.92 \%$ de proteína en Tomatlán, de 19.44 en la Cruz de Huanacaxtle, 31.79 en Autlán, 22.85 en la Huerta, todos en el estado de Jalisco. En general, en estas discrepancias pueden influir las condiciones edafológicas del sitio y diferentes períodos de estudio.

\section{Fracciones de fibra de la harina de los frutos de E. cyclocarpum y B. alicastrum.}

Con relación a las fracciones de fibra para las diferentes harinas de los frutos en estudio se muestran en el Tabla 4. Los contenidos de fibra total expresados como FDA y FDN para $E$. cyclocarpum, variaron entre 31.28 y $34.58 \%$ y para B. alicastrum 17.25, $19.37 \%$ concernientemente lo cual puede indicar que la disponibilidad de nutrientes en los frutos completos es variable, dependiendo del nivel de fibra presente.

El promedio de FDN y FDA para $E$. cyclocarpum, son superiores a los reportados por Cecconello et al., $2003(27.53,19.9)$ y Sosa $e t$ al., 2004 (41.20 y 24.83\%). Estos valores son muy parecidos a los reportados por Febles, et al., (1999), donde los estimaciones son: FDN 41.5, FDA 29.8, lignina (lig) 58, celulosa (cel) 22.5 y hemicelulosa (hemi) $11.7 \%$. Es posible que la diferencia encontrada con estos autores se deba a que estos frutos fueron evaluados en diferente país por lo tanto, diferentes condiciones climáticas y edafológicas.

Dentro de los trabajos realizados con frutos de $B$. alicastrum se encuentran los de Lizarraga et al., 2001; Sosa et al., 2004, los cuales reportaron valores de 37.5, 28.5, 67.50, $47.10,61.5,4.0$ y $43.48 \%$ en FDN y FDA, por su parte, Delgado et al., (2000), reportaron los siguientes valores; (FDN 55.8, FDA 35.8, lig 83, cel 25 y hemi 20\%), valores muy superiores a los encontrados en este trabajo.

\section{Contenido de minerales de la harina de los frutos de las arbóreas en estudio}

El contenido de minerales de la harina de $E$. cyclocarpum y B. alicastrum se observan en el Tabla i4 donde el potasio se encuentra en mayor porcentaje en el fruto de B. alicastrum con 1.00 $\%$ con respecto a la harina de E. cyclocarpum $(0.86 \%)$, sin embargo, nitrógeno $(\mathrm{N})(3.66 \%)$, se encuentra en mayor cantidad en la harina de $E$. cyclocarpum. Delgado et al., (2000), señalaron que el E. cyclocarpum contiene el uno porciento de fósforo y calcio. Sin embargo, Carranza et al., (2003) y Sosa et al., (2004) encontraron los valores de $0.9,4.51 \%, 11.7,19 \mathrm{ppm}$ para estos mismos minerales. 
En un trabajo publicado por Cecconello et al., (2003), reportaron valores de 2.26, 0.29, $0.36,0.77,0.23 \%$ y $5.55 \mathrm{ppm}$ para nitrogeno fósforo, calcio, magnesio $(\mathrm{Mg})$, azufre $(\mathrm{S})$ y cobre $(\mathrm{Cu})$. Son valores muy similares a los reportados en este trabajo, con la diferencia de que ellos evaluaron en Venezuela. Por su parte Román et al., (2004), publicaron los siguientes resultados $2.45 \%$ de N, 11.7, 19, $0.29 \mathrm{ppm}$ de $\mathrm{P}$, K y Calcio. Barrientos (2006), reportó para la harina del fruto completo de E. cyclocarpum valores de $2.21,1.59,0.81,0.32,0.19,0.27$, 0.052 y $0.034 \%$ de N, Ca, K, Mg, sodio (Na), P, $\mathrm{Cu}$ y fierro en la localidad de Tomatlán, Jalisco. Destacandose el N (3.66\%), de los reportados por los otros autores. Para B. alicastrum Carranza et al., (2003) reportaron 0.14 y $2.67 \%$ de fósforo y calcio. Román et al (2004) encontraron $1.54 \%$ de N, 4, 20, 0.65 ppm de $\mathrm{P}$, $\mathrm{K}$ y Ca respectivamente. Los valores reportados por estos autores son similares a los obtenidos en este trabajo, las diferencias pueden ser por la calidad del sitio y año de observación.

\section{Prueba de comportamiento}

El consumo de alimento diario para el tratamiento de las dietas testigo, la inclusión del 10 y el $20 \%$ de harina de los frutos de $E$. cyclocarpum y de B. alicastrum no se encontró diferencia significativa $(\mathrm{P}>0.05)$, entre $\operatorname{los}$ tratamientos, al realizar el análisis de varianza. La inclusión de los diferentes niveles de harina de E. cyclocarpum y B. alicastrum no modificó explicativamente el consumo de alimento con respecto a la ración testigo. Estos datos se pueden observar más objetivamente en el Tabla 5 .

\begin{tabular}{|l|r|r|}
\hline \multicolumn{1}{|c|}{ Tratamientos } & $\begin{array}{r}\text { E. cyclocarpum } \\
(\mathrm{g})\end{array}$ & $\begin{array}{c}\text { B. alicastrum } \\
(\mathrm{g})\end{array}$ \\
\hline $\mathrm{T}_{1} \mathrm{y}_{4}$ & $289^{\mathrm{a}}$ & $292^{\mathrm{b}}$ \\
\hline $\mathrm{T}_{2} \mathrm{y}_{5}$ & $291^{\mathrm{a}}$ & $294^{\mathrm{a}}$ \\
\hline $\mathrm{T}_{3} \mathrm{y}_{6}$ & $297^{\mathrm{a}}$ & $301^{\mathrm{a}}$ \\
\hline
\end{tabular}

$\mathrm{T}_{1} \mathrm{~T}_{4=\text { dieta testigo, }} \mathrm{T}_{2} \mathrm{~T}_{5=10 \% \text { de inclusión, }} \mathrm{T}_{3} \mathrm{~T}_{6=20 \% \text { de inclusión }}$

No hay diferencia significativa entre tratamientos $(\mathrm{P}<0.05)$

Tabla 5 Consumo de alimento diario para los ovinos en los seis tratamientos

Aún el no existir diferencias estadísticas en el consumo de las diferentes raciones en estudio, los resultados mostraron una tendencia positiva entre el consumo y el nivel de inclusión de harina de E. cyclocarpum y B. alicastrum en la dieta.
Esta tendencia difiere con los resultados obtenidos por González et al., (1989), quienes encontraron un menor consumo de materia seca al comparar un nivel de inclusión de $31.1 \%$ en contra de 0 y $15.5 \%$ de E. cyclocarpum. González et al., (1989) y Amaro et al., (1993), señalan que el máximo consumo voluntario de materia seca se encontró en los niveles de inclusión del 31 y el $48 \%$, en tanto que Bonilla, (1999), indicó que el consumo voluntario aumento conforme se incrementó la inclusión de la harina de E. cyclocarpum en la dieta de 40 a $61 \%$. Los resultados anteriores mostraron una gran variación entre la relación que guardan el nivel de inclusión de la harina de los frutos de $E$. cyclocarpum y B. alicastrum y el consumo de alimento de las diferentes raciones, este fenómeno pudiera estar explicado por los diferentes tiempos de secado del fruto y de almacenado de la harina, referidos por los distintos autores.

En este estudio, el fruto fue secado al sol durante 10 días por espacio de ocho horas, el fruto fue molido y ofertado a los animales con un periodo de acopio de seis meses, mientras que Pérez y Zapata, (1993), utilizaron ocho días de exposición y un período corto de almacenaje, en tanto Alvarez et al., (2003), utilizaron la harina de E. cyclocarpum después de un año de almacenaje. Este periodo de almacenaje pudo influir en algunas de las características organolépticas del alimento modificando su consumo, tanto en el E. cyclocarpum como el $B$. alicastrum.

\section{Aumento de peso}

En cuanto al aumento de peso diario para los diferentes tratamientos, se observó diferencias significativas $(\mathrm{P}<0.05)$.

\begin{tabular}{|l|r|r|}
\hline Tratamientos & $\begin{array}{r}\text { E. cyclocarpum } \\
(\mathrm{g})\end{array}$ & $\begin{array}{c}\text { B. alicastrum } \\
(\mathrm{g})\end{array}$ \\
\hline $\mathrm{T}_{1} \mathrm{y} \mathrm{T}_{4}$ & $242^{\mathrm{a}}$ & $230^{\mathrm{a}}$ \\
\hline $\mathrm{T}_{2} \mathrm{y}_{5}$ & $220^{\mathrm{a}}$ & $238^{\mathrm{a}}$ \\
\hline $\mathrm{T}_{3} \mathrm{y}_{6}$ & $167^{\mathrm{b}}$ & $198^{\mathrm{b}}$ \\
\hline
\end{tabular}

$\mathrm{T}_{1} \mathrm{~T}_{4=\text { dieta testigo, }} \mathrm{T}_{2} \mathrm{~T}_{5=10 \% \text { de inclusión, }} \mathrm{T}_{3} \mathrm{~T}_{6=20 \% \text { de inclusión }}$ Letras diferentes en lineas indican diferencia significativa $(\mathrm{P}<0.05)$

Tabla 6 Aumento de peso diario de los ovinos en los diferentes tratamientos 
Los resultados de González et al., (1989), mencionaron ganancias de peso diario de 96 a $102 \mathrm{~g}$ con niveles de inclusión de 15 y $30 \%$ de harina de E. cyclocarpum, en raciones basadas en 73 y $63 \%$ de rastrojo de maíz respectivamente. Pérez et al., (1991), encontraron $101 \mathrm{y} 141 \mathrm{~g}$ de aumento de peso con inclusiones de 10 y $20 \%$ de la harina cuando proporcionaron $40 \%$ de rastrojo de maíz y $20 \%$ de sorgo, con animales criollos y de un peso inicial de $22 \mathrm{~kg}$. Bonilla, (1999), reportó que cuando se utilizaron niveles de 12 y $24 \%$ de inclusión de harina de E. cyclocarpum, en una dieta con un contenido de $40 \%$ de rastrojo de maíz y $14 \%$ de grano de sorgo obtuvo un aumento de peso de $127 \mathrm{~g} /$ día.

Álvarez et al., (2003), en un trabajo realizado con harina de E. cyclocarpum y gallinaza con inclusiones del 15 y el $30 \%$ obtuvo una ganancia de peso diaria de 86 y 74 g. Por otro lado Peralta et al., (2004) reporta 168, 160, y $125 \mathrm{~g}$ de aumento de peso con niveles de inclusión de harina de E. cyclocarpum de 10, 20 y $30 \%$. Como podemos observar en este trabajo los aumentos de peso fueron superiores a los reportados por otros autores, mientras que las tablas de nutrición (NRC, 1996), recomienda el $16 \%$ de proteína para animales confinados, de razas especializadas, con un promedio de 20 a 25 $\mathrm{kg}$ de peso, y para ganancia de peso de 250 y 300 g por día, valores muy similares a los obtenidos en este trabajo.

Conversión alimenticia. En la conversión alimenticia, se observan diferencias significativas $(\mathrm{P}<0.05)$ para el tratamiento de la inclusión del $20 \%$ de harina de E. cyclocarpum y de $B$. alicastrum en relación con los demás tratamientos (Tabla 7)

\begin{tabular}{|l|c|c|} 
Tratamientos & $\begin{array}{c}\text { E. } \\
\text { cyclocarpum } \\
(\mathrm{kg})\end{array}$ & $\begin{array}{c}\text { B. } \\
\text { alicastrum } \\
(\mathrm{kg})\end{array}$ \\
\hline $\mathrm{T}_{1} \mathrm{y} \mathrm{T}_{4}$ & $6.13^{\mathrm{b}}: 1$ & $6.51^{\mathrm{b}}: 1$ \\
\hline $\mathrm{T}_{2} \mathrm{y}_{5}$ & $6.78^{\mathrm{b}}: 1$ & $6.36^{\mathrm{b}}: 1$ \\
\hline $\mathrm{T}_{3} \mathrm{y}_{6}$ & $7.05^{\mathrm{a}}: 1$ & $7.81^{\mathrm{a}}: 1$ \\
\hline
\end{tabular}

$\mathrm{T}_{1} \mathrm{~T}_{4=\text { dieta testigo, }} \mathrm{T}_{2} \mathrm{~T}_{5=10 \%}$ de inclusión, $\mathrm{T}_{3} \mathrm{~T}_{6=20 \% \text { de }}$ inclusión

Tabla 7 Conversión alimenticia para los ovinos en los diferentes tratamientos

Letras diferentes en columnas hay diferencia significativa $(\mathrm{P}<0.05)$
La conversión alimenticia de los animales en todos los tratamientos con inclusión de harina de fruto de E. cyclocarpum, fue mejor a la encontrada por Bonilla (1999), quien al utilizar una inclusión de $48 \%$ en la ración obtuvo una conversión alimenticia de 8:1 (es decir que necesita ocho unidades de alimento para producir una de carne).

Por otro lado Álvarez et al., (2003) utilizando $30 \%$ de harina de E. cyclocarpum y hasta un $20 \%$ de rastrojo de maíz en la dieta obtuvo una conversión de 7.47:1.

Peralta et al, (2004), en un trabajo realizado con ovinos estabulados y utilizando la inclusión de 10, 20 y $30 \%$ de E. cyclocarpum, encontró que la conversión alimenticia fue estadísticamente similar para todos los tratamientos (4.7:1, 3.9:1 y 4.7:1), superior al encontrado en esta investigación.

Finalmente Pérez et al., 1991, mencionaron que con la inclusión de $20 \%$ de harina de frutos de E. cyclocarpum, se consiguió la mayor conversión alimenticia (6.98) y el menor costo de producción por concepto de alimentación (\$2.20), resultando similar al encontrado en este trabajo.

\section{El costo estimado de alimento por kilogramo de carne producida}

Para los tratamientos estudiados en ovinos en estabulación, lo podemos observar en el (Tabla 8), el costo más bajo correspondió a la inclusión del $10 \%$ de E. cyclocarpum, y el más alto, para la dieta testigo. Los costos de la alimentación decrecieron, en un 14 a un $26 \%$ por $\mathrm{kg}$ de carne producida, comparado con la ración testigo, (Tabla 8).

Se calculó el costo por kilogramo de la harina de los frutos de E. cyclocarpum y de $B$. alicastrum considerando el salario mínimo de la localidad aplicado a mano de obra para recolección, molienda e integración a los tratamientos estudiados. 


\begin{tabular}{|c|c|c|c|c|c|c|}
\hline & Testigo & E.cyclc & carpum & B. alic & astrum & $\begin{array}{c}\text { Costo/kg } \\
\text { de } \\
\text { ingrediente }\end{array}$ \\
\hline Ingredientes & & $10 \%$ & $20 \%$ & $10 \%$ & $20 \%$ & \\
\hline \begin{tabular}{|ll}
$\begin{array}{l}\text { Rastrojo } \\
\text { maíz }\end{array}$ & $\mathrm{de}$ \\
\end{tabular} & 27.00 & 54.50 & 27.00 & 54.50 & 27.00 & 1.65 \\
\hline Alfalfa & 12.00 & - & 17.40 & - & 13.20 & 2.55 \\
\hline Trigo & 22.00 & - & 4.80 & - & 4.80 & 2.85 \\
\hline \begin{tabular}{|l|} 
Sorgo \\
\end{tabular} & 20.00 & 17.00 & 15.00 & 15.00 & 15.00 & 2.35 \\
\hline Melaza & 14.50 & 10.00 & 10.00 & 10.00 & 10.00 & 2.00 \\
\hline $\begin{array}{|ll|}\begin{array}{l}\text { Harina } \\
\text { pescado }\end{array} & \mathrm{de} \\
\end{array}$ & 2.00 & 3.20 & 1.00 & 5.50 & 5.20 & 8.00 \\
\hline Harina de frutos & - & 10.00 & 20.00 & 10 & 20 & 2.00 \\
\hline Roca fosfórica & 1.00 & 1.00 & 1.00 & 1.00 & 1.00 & 1.40 \\
\hline \begin{tabular}{|l|}
$\begin{array}{l}\text { Carbonato } \\
\text { calcio }\end{array}$ \\
\end{tabular} & 1.00 & 1.00 & 1.00 & 1.00 & 1.00 & 3.80 \\
\hline Vitaminas* & 0.25 & 0.25 & 0.25 & 0.25 & 0.25 & 9.00 \\
\hline Minerales* & 0.05 & 0.05 & 0.05 & 0.05 & 0.05 & 0.95 \\
\hline Sal & 1.00 & 1.00 & 1.00 & 1.00 & 1.00 & 1.45 \\
\hline Urea & 1.60 & 2.00 & 1.50 & 2.00 & 1.50 & 4.30 \\
\hline Totales & 100.00 & 100.00 & 100.00 & 100.00 & 100.00 & \\
\hline $\begin{array}{|ll|}\text { Costo/kg } & \mathrm{de} \\
\text { Alimento } & \\
\end{array}$ & 2.45 & 2.13 & 2.21 & 2.42 & 2.44 & \\
\hline
\end{tabular}

Tabla 8 Porcentaje de los Ingredientes de las raciones en estudio

\begin{tabular}{|c|c|c|c|}
\hline Tratamientos & $\begin{array}{l}\text { Conversión } \\
\text { alimenticia }\end{array}$ & $\begin{array}{l}\text { Costo por } \\
\mathrm{kg} \text { de } \\
\text { ración }\end{array}$ & $\begin{array}{c}\text { Costo alimento / } \\
\text { kg de carne } \\
\text { producido }\end{array}$ \\
\hline \multicolumn{4}{|c|}{ E. cyclocarpum } \\
\hline $\mathrm{T}_{1}$ & $6.36^{\mathrm{a}}: 1$ & 2.80 & 17.80 \\
\hline $\mathrm{T}_{2}$ & $6.78^{a}: 1$ & 2.29 & 15.52 \\
\hline $\mathrm{T}_{3}$ & $7.05^{\mathrm{b}}: 1$ & 1.81 & 12.76 \\
\hline \multicolumn{4}{|r|}{ B. alicastrum } \\
\hline $\mathrm{T}_{4}$ & $6.51^{\mathrm{a}}: 1$ & 2.80 & 18.23 \\
\hline $\mathrm{T}_{5}$ & $6.13^{a}: 1$ & 2.35 & 14.41 \\
\hline $\mathrm{T}_{6}$ & $7.81^{\mathrm{b}}: 1$ & 2.04 & 15.93 \\
\hline
\end{tabular}

$\mathrm{T}_{1} \mathrm{~T}_{4=\text { dieta testigo, }} \mathrm{T}_{2} \mathrm{~T}_{5=10 \%}$ de inclusión, $\mathrm{T}_{3} \mathrm{~T}_{6=20 \% \text { de }}$ inclusión

Tabla 9 Conversión alimenticia y costo estimado en los diferentes tratamientos en ovinos en estabulación

El costo del kilogramo de carne producida el más bajo, correspondió al $10 \%$ de inclusión de harina de E. cyclocarpum, aunque este tratamiento no fue el mejor en cuanto a consumo de alimento y conversión alimenticia, esto se puede deber a que la fuente proteica de la ración es la de mayor costo, y con esta inclusión del $10 \%$ se bajan los costos de producción. Peralta et al., (2003), mencionaron que el costo de alimento por carne producida en ovinos en estabulación, para la dieta testigo fue de $\$ 10.76$, en las inclusiones del 10, 20 y 30 de harina de frutos de E. cyclocarpum fue de; 9.35, $7.21 \mathrm{y}$ 8.37 respectivamente, sus costos fueron menores a los encontrados en este trabajo, este fenómenos se puede deber a que no le asignaron un costo a la harina de los frutos o que la fecha de la investigación fue tres años anteriores a este trabajo, porque los costos de los insumos aumentan considerablemente año con año.

\section{Conclusiones}

La inclusión de harina de los frutos de $E$. cyclocarpum y de B. alicastrum en las raciones alimenticias de los ovinos mejoró el consumo. El consumo de alimento fue mejor con la inclusión del $10 \%$ de B. alicastrum. La conversión alimenticia y el aumento de peso fue mejor para la dieta testigo $\mathrm{T}_{1}$.

El valor económico de la ración alimenticia con la inclusión de las harina de los frutos de E. cyclocarpum, el mejor fue con el $10 \%$, como sustituto de una fuente proteica, en ovinos estabulados, lo que permitió mantener su desarrollo con una disminución significativa de los costos de producción. Hay que considerar que el costo se calculó con el de la mano de obra local y no puede aplicarse a nivel nacional o internacional.

La inclusión de harina de los frutos de E. cyclocarpum y de B. alicastrum en la alimentación de rumiantes es una alternativa técnicamente viable, dependiendo del nivel de inclusión, la distancia y los costos de transporte a las plantas de producción.

la respuesta productiva encontrada en los ovinos suplementados con frutos de leguminosas arbóreas está asociada principalmente con aumento en el consumo voluntario de materia seca y energía digestible, mayor flujo de proteína microbial al duodeno y un mejor balance entre nutrientes gluco y cetogénicos

\section{Referencias}

Álvarez, M; Melgarejo, M. y Castañeda, N. 2003. Ganancia de peso, conversión y eficiencia alimentaria en ovinos alimentados con fruto (semilla y vaina) de E. cyclocarpum (Enterolobium cyclocarpum) y pollinaza. Vet. Méx. 34(1) 2003 p 34

Amaro, G R, Bonilla, C J A. y Llamas, L G. 1993. Consumo voluntario y digestibilidad in vivo de dietas con inclusión de vaina de guanacastle en ovinos. Memorias de la Reunión Nacional de Investigación Pecuaria. Guadalajara, Jalisco, México. pp130

AOAC 1990. Official Methods of Analysis. Association of Official Anallytical Chemists, $5^{\text {ed }}$ (P A Cunnif, ed.). AOAC International Arlington. p 2000 
Barrientos, R. L. 2006. Variación en la composición protéica de la semilla madura de parota (Enterolobium cyclocarpum Jacq. Griseb) en el centro - occidente de México. Tesis de Doctor en Ciencias Àrea de Écología, CUCBA, Univ. de Guad. pp 42- 47

Bondi, A. 1988. Nutrición Animal. Editorial Acribia, S.A. Zaragoza, España. pp.544

Bonilla, C.J. 1999 Uso de vainas de parota en dietas para finalización de ovinos. En 500 Tecnólogias Llave en Mano. Primera Edición. SAGAR-INIFAP. México, D.F, p 20

Carranza, M M; Sanchez V L; Pineda, L R; Cuevas, G R; 2003. Calidad y potencial forrajero de especies del bosque tropical caducifolio de la sierra de Manantlan, México. Agrociencia, marzo-abril, No, 02. Colegio de Posgraduados, Texcoco, Mexico. pp 206-207

Cecconello, C; Benezra, M; Obispo, N. 2003. Composición quimica y degradabilidad ruminal de los frutos de algunas especies forrajeras leñosas de un bosque seco tropical. Zootecnia Tropical 21(2), pp 149 - 165

Delgado, Denia; C, O, La O, Bertha, Chongo, Juana, Galindo y Yusleidys, Santos 2000. Determinación del valor nutritivo del follaje de dos árboles forrajeros tropicales: Brosimun alicastrum y Bauhinia galpinii. IV Taller Internacional Silvopastoril. Los árboles y arbustos en la ganaderia tropical 29 de noviembre-1ro. De diciembre del 2000 Tomo 1. Estación experimental de pastos y forrajes "Indio Hatuey" Cuba. pp 102 - 104

Enkerlin, E.C., Cano, G., Garza, R.A. Y Vogel, E. 1997. Ciencia Ambiental y Desarrollo Sostenible. International Thomson Editores. México, D.F. p. 666

Febles, G.; Ruiz, T.E; Chongo, J.; Alonso. O; I. Scull, H. Gutierrez, D. y Hernández, L. 1999. Evaluación de diferentes especies de árboles y arbustos para el desarrollo de sistemas silvopastoriles en el trópico. Memorias del primer congreso latinoamericano de agroforesteria para la producción animal sostenible. Cali, Colombia. pp 1 - 9
Giraldo, L. A. 1996a. Efecto de tres Densidades de Árboles en el Potencial Forrajero de un Sistema Silvopastoril Natural. IN: Memorias Seminario Internacional Sistemas Silvopastoriles: Casos Exitosos y su Potencial en Colombia. Santafé de Bogotá, La Dorada, Santa Marta: Noviembre 27-29/diciembre 1996. Ministerio de Agricultura y Desarrollo Rural pp $57-72$

González, SA, Ariceaga N, Altamirano A y Huerta M 1989. Evaluación del valor nutritivo de la parota (Enterolobium cyclocarpum) en los alimentos de ovinos. Memorias del Segundo Congreso Nacional de Producción Ovina. San Luis Potosí Mexico. pp 113-115

Laker, M.W. 1994. Where is the beef?: Incorporating cattle into sustainable agroforestry systems in the Amazon Basin. Agroforestry Systems. 25. pp 227-241

Lizarraga, S. H., Solorio, S. F.J., Sandoval, C. C.A. 2001. Evaluación agronómica de especies arbóreas para la producción de forraje en la Peninsula de Yucatán. Livestock Research for Rural Development 13(6)2001. pp 1 -9

National Reserarch Council. (1996). Nutrient requirements of dairy cattle. Sixth edition p 34

Palma, J.M. 2006. Los sitemas silvopastoriles en el trópico seco mexicano. Arch. Latinoam. Prod. Anim. 2006. 14 (3). pp 95 - 104

Peralta N., Palma, JM., Macedo, R. 2004. Efectos de diferentes niveles de inclusión de parota (Enterolobium cyclocarpum) en el desarrollo de ovinos en estabulación. FMVZ. Universidad de Colima. Tecomán, Colima. p 3

Pérez, E; Perezgrovas, R y Sosa, R. 1991. Utilización de frutos de guanacaste ( $E$. cyclocarpum Jacq. Griseb) como fuente de proteina en la alimentación de borregos. Memorias del Cuarto Congreso Nacional de Producción Ovina. Chiapas, Mexico. pp 10 - 11

Pérez, R.J y Zapata, B.G. 1993 Utilización de ramón Brosimun alicastrum en alimentación de ovinos en crecimiento. In Reunion Nacional de Investigación Pecuaria (Puerto Vallarta, Mex.). Memorias., S.N.T. p 157 
Román, L.M; Mora, S. A; Gallegos, R. A. 2004. Especies arboreas de la costa de Jalisco, Mexico, utilizadas como forraje en sistemas silvopastoriles. Scientia - CUCBA 6(1-2); 3 11.2004 p 10

Sosa R. E.E., Perez R. D., Ortega R. L., Zapata B. G. 2004. Evaluación del potencial forrajero de árboles y arbustos tropicales para la alimentación de ovinos. Tec. Pecu. Mex. 2004:42 (2) pp 129 - 144

Sotelo, A., Contreras, E. and Flores,S. 1997. Nutritional value and content of antinutritional compounds and toxics in ten wild legumes of Yucatan Peninsula. Plant Foods for Human Nutrition. pp 115-123

Van Soest, P y R. Wine. 1967. Uses of detergent in the analysis of fibrous forages. IV Determination of plant cell-wall constituents. J. Assoc. Off. Anal. Chem. 50 pp 50-55

Van Soest, P.J. 1979. Physico-chemical aspects of fibre digestion. In: Digestion and metabolism in the ruminant (eds. I. W. McDonald and A. C. I. Warner), p. 351-356 